\title{
Bias due to individual differences in yoked control designs
}

\author{
H. D. KIMMEL AND F. R. TERRANT, OHIO UNIVERSITY, \\ Athens, Ohio
}

Church's objections to the yoked control design are reviewed and criticized. To deal with them more effectively, a reciprocal yoked control design is presented, in which each $S$ of a yoked pair receives training with two CSs, one of which involves an instrumental contingency while the other is in a yoked control arrangement with the other S's instrumental CS. Thus, each $S$ in the pair serves as a yoked control for the other, in reciprocal relation to the two CSs used. In addition to eliminating inadvertent confounding stemming from individual differences in sensitization and responsiveness, the reciprocal yoked control design also provides a single-session method for identifying $S$ who are equally classically conditionable, so that instrumentalclassical comparisons can be made free of bias from differences in classical conditionability. In addition, this design provides a means for testing the empirical soundness of Church's arguments regarding differences in conditionability and biased outcomes. Methods for statistical analysis of the reciprocal yoked control design are also considered.

In experiments involving response-contingent presentations of appetitive or aversive reinforcing events, a yoked control design is frequently used to ensure that observed changes in behavior can be attributed to the experimental response-reinforcement contingency. Subjects (Ss) are paired in such a way that the behavior of one, the "experimental" $S$, determines whether both Ss receive the event. Within each pair assignment as "experimental" or "control" is random. There is no requirement that the two Ss actually be run simultaneously, although, in experiments in which the temporal occurrence of the reinforcing event depends upon when the response occurs, simultaneous data-collection may be necessary. The yoked control design provides both Ss with the same number and trial-by-trial (or minute-by-minute) pattern of stimuli and, seemingly, permits only the effect of the responsereinforcement contingency to differentiate them.

As Church (1964) has observed, the conventional yoked control design may entail inadvertent confounding, resulting in more frequent rejection of true null hypotheses than is indicated by stated probabilities. This would be the case, he argues, if the Ss tend to differ from one another in their reactions to features of the reinforcing event other than its close temporal continguity to the response. For example, Kimmel \& Baxter (1964) used yoked control Ss in their study of avoidance conditioning of the GSR. The avoidance $S$ and his yoked control each received paired CSs and shocks on only those trials on which the avoidance $S$ failed to make a criterion $C R$. On trials on which a criterion $C R$ was made by the avoidance $S$, both $S$ s in the yoked pair received only the CS. According to Church, if the Ss in a yoked pair differ in classical conditionability (or reactivity, etc.), this difference will have an asymmetrical effect depending upon which of the two Ss is the better classical conditioner (or the more reactive, etc.). If the avoidance $S$ is the better classical conditioner, both Ss will receive few shocks and, in particular, the control $S$ will not receive enough shocks to reach a high level of classical conditioning. If the control $\mathrm{S}$, however, is the better classical conditioner, both Ss will receive many shocks, most of which will be wasted on the control $\mathrm{S}$ who would quickly achieve maximal classical conditioning. Thus, a difference in favor of the avoidance procedure over the yoked control procedure would spuriously result even if avoidance conditioning of the GSR were impossible.

The purpose of this paper is to describe a new yoked control design which permits this source of contamination to be dealt with effectively. It is desirable to elucidate the most significant features of this new design even while serious reservations regarding Church's arguments are registered. The latter disclaimer is entered to make certain that our presentation of a design intended to overcome Church's objections to previous yoked control designs in no way is interpreted as agreement with his arguments. In fact, two basic assumptions implicitly made by Church seem to us to be all but untenable from what is now known about classical conditioning. Church assumes, first, that the avoidance $S$ who happens by chance to be a better classical conditioner than his yoked control will rise very quickly to a high level of conditioning and, thereafter, receive no further shocks. While this possibility may exist in situations involving a discrete, skeletal response ${ }^{1}$, it is certainly far from what happens in the case of autonomically mediated responses such as the GSR. Omission of the shock in classical GSR conditioning rather quickly results in extinction and, in an avoidance study, a need for more shocks. This would still result in somewhat fewer shocks being given to the control $S$ than he may need, but the possible error is of greatly lesser magnitude than implied by Church. The second questionable assumption made by Church is that the many shocks received by the control $S$, when he is the better conditioner of the pair, will not continue to benefit him after he quickly reaches asymptotic performance. In situations in which magnitude of response is the main dependent variable, as opposed to response frequency, this assumption may be quite unrealistic. While it may be true that the response begins to occur early in

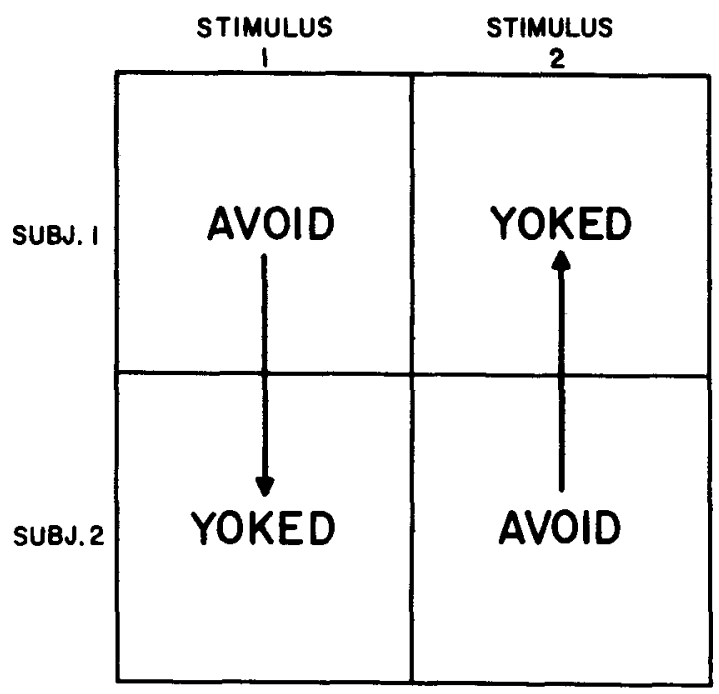

Fig. 1. Experimental plan for a study of avoidance conditioning, using the reciprocal yoked control design. Ss are assigned at random to the rows and stimuli to the columns. 
training and may continue to occur thereafter, its size has considerable opportunity to keep growing with further paired trials.

\section{RECIPROCAL YOKING}

In the reciprocal yoked control design $S$ s are run in pairs as in conventional yoked designs. However, instead of using a single CS with an avoidance (or punishment, etc.) contingency for one $S$ with the other $S$ yoked, the new design uses two CSs. The behavior of one of the $S$ s determines whether the reinforcing event is delivered to both Ss in conjunction with one of the CSs, while the behavior of the other $S$ determines whether the reinforcing event is delivered to both $S$ s in conjunction with the other CS. Thus, each $S$ in the pair serves as a yoked control for the other, in reciprocal relation to the two CSs involved.

Individual differences in sensitization tendencies or general reactivity are completely eliminated as a source of bias in this design. since both $\mathrm{Ss}$ in a yoked pair receive the same total number of shocks. However, if one of the Ss is a better classical conditioner than the other, an asymmetrical influence of the type Church has discussed might still be expected to enhance that $S$ 's relative performance in response to his avoidance CS (as compared with the other S's performance in response to that CS). but not his relative performance in response to the other $S$ 's avoidance CS (as compared with the other S's performance in response to that $(S)$. In other words, few shocks would be delivered with the better S's avoidance CS. making it unlikely that the poorer $S$ would achieve a high level of conditioning to that (S. Many shocks would have to be given with the poorer S's avoidance CS. but these would not benefit the better conditioner after he had reached asymptotic performance. At best. the poorer conditioner might achicve a level of responding on his avoidance ( $S$ that spproached the better conditioner's performance on that ( $S$. The net avoidance-control comparison in a yoked pair, thus. could still contain the spurious error favoring the avoidance condition. since each pair of $\mathrm{Ss}$ can be conceptualized as representing the two types of asymmetrical pairs that Church has culplasized.

The advantage of reciprocal yoking with respect to the foregoing problem. lies in the fact that, if one of the $S s$ in a pair is a better conditioner than the other (which. in general, may assumed to always be somewhat the case). the avoidance-control contrast may he evaluated in the data of only those pairs of Ss in which Churclis bias is absent. For example. if any bias is present in the avoidance-control differences of the better conditioning Ss. it should surely not favor the avoidance CS, since relatively fewer shocks would have been presented with the avoidance CS than with the other CS (the latter being determined by the poorer conditioner). The determination of which $S$ is the better classical conditioner in each pair may be made objectively on the basis of the number of shocks delivered with each S's avoidance CS. following quite faithfully linurch's reasoning that the number of shocks nceded with the avoidance $\mathrm{CS}$ is an index of classical conditionability (Church's arguments rest on the assumption that a better classicul conditioner would "need" fewer shocks). On the basis of a null hypothesis of equal proportions of shocked trials for the two Ss. Fisher's exact test for a fourfold contingency table could be used to select those Ss who are significantly better conditioners than their yoked controls This procedure can also be used to identify those pairs of Ss who do not differ in conditionability (who received the same or almost the same number of shocks on each stimulus). This would make it possible to implement Church's suggestion that Ss who are matched in conditionability be used in yoked pairs. The reciprocal yoked control design has the virtue of providing these essential data on classical conditionability without the necessity of employing a preliminary classical conditioning session. As has been noted elsewhere (Kimmel, 1967), preceding avoidance conditioning by a classical conditioning procedure is quite likely to reduce the probability that avoidance conditioning can occur.

In addition to providing selected samples of $S s$ in which unbiased (or negatively biased) comparisons of the avoidance and control conditions could be made, the reciprocal yoked design also permits a test to be made of Church's principal proposition, that individual differences do, in fact, contribute to observed differences in yoked control studies. Given that the two Ss in a yoked pair will almost never be exactly equally good conditioners, it follows that the average degree of observed avoidancecontrol difference in a pair would vary as a function of the difference in classical conditionability between the two Ss. That is, a test may be nuade of the hypothesis that the correlation between the average within-pair avoidance-control differences and the differences between the number of shocks each $S$ in a pair received with his avoidance $C S$ does not differ from zero.

Prior to considering methods of statistical analysis, it is appropriate to comment on the desirability of shifting to what is essentially a within-S design in research that has typically employed a between-S methodology. From the point of view of the "purist," it might be argued that avoidance conditioning, say, of the eyelid reflex or the GSR should be demonstrable in both between- and within-S designs, if it is a phenomenon of any significance whatsoever. We know. however, that differential conditioning within-S may be attenuated by stimulus generalization of both excitatory and inhibitory tendencies. which may both increase responding to the negitic CS and reduce responding to the positive CS. With respect to possible influences of such generalization. the within-S design would be expected to reveal weaker effects than are shown in between-S designs (this should also reduce within-S bias). On the other hand, a kind of "contrast" phenomenon in within-S designs has recently been reported (Grice \& Hunter. 1964), in which greater within-S differences in responding to two stimuli are found than in between-S comparisons. It would be almost impossible in advance of data-collection. to predict which of these two possible (and opposed) influences would operate in a particular study (or. if both were present, which would prevail). Comparisons of the between-S avoidance-control differences in the reciprocal yoked design with those found in conventional, single CS, yoked studies would identify at least the direction of influence that may result from shifting to the within-S method.

Concern regarding possible generalization or contrast effects in the within-S reciprocal yoked control design would appear to imply that two CSs of the same sensory modality be used. e.g., two visual stimuli of different shapes and/or spatially separated. This would also reduce the likelihood that individual differences in differential reactivity to stimuli of different sense modalities would inte"act with which stimulus happened to be which S's avoidance stimulus and which his control stimulus. Such an interaction could also produce systematic bias favoring the avoidance condition. But, it is far less likely that Ss would differ in their differential reactivity to, say, visual stimuli on the right and left, or visual stimuli of different shapes, than they would to 
visual vs auditory stimuli. Using two stimuli of the same sensory modality also eliminates the unnecessary complications of possible intersensory dominance.

\section{STATISTICAL ANALYSIS}

The basic plan of a reciprocal yoked control design for a study of avoidance conditioning is presented in Fig, 1. The figure illustrates the simple Latin square arrangement of the two yoked Ss and the two CSs, the experimental treatment (avoidance vs control) being represented as the Latin square factor. Our earlier concern regarding the possibility that the two Ss may react differentially to the two CSs, in different ways, is justified by the observation that, as in any 2 by 2 Latin square, the row-column interaction is completely confounded with the avoidance-control degree of freedom on the face of the square. However, as has been noted already, the likelihood of such an interaction actually existing is reduced to negligibility by using two stimuli of the same modality.

Ignoring the fact that each $\mathrm{S}$ determines the number of shocks received by the other $S$ for one of the two CSs (and that these numbers may differ if the Ss differ in classical conditionability, which is Church's main point), CR data from an experiment of this type can be analyzed in the manner of Lindquist's Type 2 mixed factorial design (Lindquist, 1953). This should be clear when one notes that all of the Ss for which $C S_{1}$ is the avoidance stimulus and $\mathrm{CS}_{2}$ the control stimulus can be thought of as comprising Group I and all of the Ss for which the opposite arrangement holds as comprising Group 11. Since there are only two levels of each factor (avoidance vs control and $\mathrm{CS}_{1}$ vs $\mathrm{CS}_{2}$ ), their interaction is completely between-S, as has been noted in somewhat different terminology previously. The avoidancecontrol and $\mathrm{CS}_{1}-\mathrm{CS}_{2}$ contrasts, however, are both within-S effects. An earlier representation of essentially the same analysis has also been given by Grant (1949). It must be reiterated that these methods are appropriate only when one ignores the possibility that differences in conditionability between the two yoked Ss may result in different numbers of shocks being associated with the two CSs. Either Lindquist's or Crant's methods could be used, of course, on data of yoked pairs within which the number of shocks associated with the two CSs was the same.

A variation of a cross-over design (Cochran \& Cox, 1957) may be used to deal more directly with the difference in classical conditionability. Given that an objective criterion exists for determining which $S$ of a pair is the better classical conditioner (such as the Fisher test mentioned above) and which is the poorer, the data of the better conditioners may be arbitrarily put in the top row and the data of the poorer conditioners in the bottom row of a statistical computation table. In this way, the single degree of freedom for Rows permits isolation of that portion of the total variation arising from within-pair differences in conditionability. But, as Cochran and Cox point out, the cross-over design deals only with the average effect of differences in classical conditionability. Since the difference in classical conditionability is likely to differ substantially from pair to pair, a modification of the cross-over design, also described by Cochran \& $\operatorname{Cox}$ (1957, pp. 128-129), may be more appropriate in the situation presently being considered. In the modified design, location of better and poorer $S$ s in the two rows of the table is randomized after data-collection and one degree of freedom for Rows is extracted for each Latin square (cach yoked pair) in the experiment. This method isolates variation due to differences in conditionability in general, as well as fluctuations in this difference from square to square. The degrees of freedom for Rows within-squares, however, come out of those previously available for error (in the crossover design). For this reason, a somewhat larger number of replications may be needed to provide an adequate error term, since there will be a total of only $s-1$ degrees of freedom for error, with $s=$ number of squares (or $\mathrm{n} / 2$ ).

A test of Church's assumption that differences in classical conditionability will result in spurious avoidance conditioning effects may also be made via an F-ratio of Rows within squares over error. Since different pairs of Ss will receive different combinations of shocks with the two CSs, the mean square for Rows within squares estimates the variability resulting from these differences (plus error variance). If this $F$ test fails to achieve significance (and if differences in number of shocks with the two CSs were present), this aspect of Church's argument would not be supported. In fact, a nonsignificant (and very small) F might be taken as support for employing the more familiar analyses mentioned earlier, with the greater number of degrees of freedom for error that they provide. In this case, an even simpler procedure would be to reduce the data in each Latin square to the average difference between avoidance and control conditions within each square and do a $t$ test of the hypothesis that the mean of these differences equals zero. As in the modified cross-over design, however, the number of degrees of freedom for this test is only one less than the number of squares.

\section{CONCLUDING REMARKS}

We have attempted, in this paper, to show that a variation in the yoked control design (referred to as reciprocal yoking) is available that deals, with varying degrees of effectiveness, with the problems recently attributed by Church (1964) to this design In particular, the reciprocal yoked control design we have described completely eliminates possible bias arising from individual differences in general reactivity or tendency toward sensitization. In addition, the design permits individual differences in classical conditionability to be dealt with more effectively than is possible using the conventional yoked design. Using a reciprocal yoked control design for data-collection and a modification of the crossover design for data-analysis, it seems possible even to isolate the source of bias of greatest concern to Church and to evaluate its actual influence. Among its other advantages, the reciprocal yoked control design also provides an objective method for identifying "better" and "poorer" classical conditioners within yoked pairs, without the necessity of preliminary classical conditioning trials.

The reciprocal yoked control design may be extended, with substantial benefit, to concomitant studies of avoidance and punishment training (or of differential reinforcement for low and high rate responding). For example, a study is now under way in our laboratory, in which reciprocal yoking is used to study avoidance conditioning in one group of $S$ s, punishment training in another group, and, in addition, two groups of Ss are being run in which both avoidance and punishment contingencies are associated with the different stimuli within each yoked pair ${ }^{2}$. In the first of these groups, one $S$ las an avoidance contingency on one CS and a punishment contingency on the other CS. The yoked control is "yoked" with respect to both CSs. In the second of these groups, one $S$ has an avoidance contingency on one of the 
CSs and serves as a yoked control for the other CS. The other S has a punishment contingency on one $\mathrm{CS}$ and serves as a yoked control for the other CS. The complete package of these four experimental cases permits Church's bias to be studied in all of its possible permutations, since careful analysis shows that it may contribute to an observed effect via a single cell of the four-fold table in the first two cases mentioned above, via two cells additively in one of the latter two cases (the last), and via none of the four cells in one of the cases (the third). Additional applications in other research areas may prove even more fruitful. REFERENCES

CHURCH, R. M. Systematic effect of random error in the yoked control design. Psychol Bull, 1964, 62, 122-131.

COCHRAN, W. G. \& COX, GERTRUDE, M. Expenimental designs New York: Wiley, 1957.

GRANT, D. A. The statistical analysis of a frequent experimental design. Amer. J. Psychol, 1949, 62, 119-122.

GRICE, G. R., \& HUNTER, J. J. Stimulus intensity effects depend upon the type of experimental design. Psychol Rev., 1964, 71, 247-256.

$\mathrm{KIMMEL}, H$. D. Instrumental conditioning of autonomically mediated behavior, Psychol Bull., 1967, 67, 337-345.

KIMIMIL, H. D., \& BAXTER, R. Avoidance conditioning of the GSR. $J$. exp. Psychol, 1964, 68, 482-485.

LINDQUIST, F. F, Design and analysis of experiments in psychology and education. Boston: Houghton-Mifflin, 1953.

MOORE, J. W., \& GORMEZANO, I. Yoked comparisons of instrumental and classical eyelid conditioning. J. exp. Psychol, 1961, 62, 552-559. NOTES

1. Even in studies using skcletal responses such as the eyelid reflex there is no assurance that the performance level achieved by avoidance Ss will be so high as to preclude conditioning of yoked controls (Moore \& Gormezano, 1961 ).

2. This study is the second author's Ph. D. dissertation research.

\section{Free association, free recall, and stimulated recall compared'}

EDWARD A. BILODEAU, TULANE UNIVERSITY, NeW Orleans, Louisiana, AND PAUL W. FOX, UNIVERSITY OF MINNESOTA, Minneapolis, Minnesota

Three primary methods of recall (free association, free recall, stimulated recall) and two modifications of them (modified free association, modified stimulated recall) were defined by use of three variables: (a) presence or absence of prior laboratory training, (b) presence or absence of $E$ controlled test stimulation, and (c) test instructions to free associate or to recall. Data obtained from approximately 300 Ss and an earlier set of 600 revealed the differential effectiveness of all three primary methods in recall and misrecall.

Behaviors of free association and retention share considerable variance (Bilodeau, 1966: Bilodeau \& Howell, in press). Until recently it was commonplace for word association and wordrecall behavior to be treated as if they were only weakly related: no study has yet been performed which provides a systematic evaluation of the communalities and differences among what appear to be three major ways of looking at the problem of word retrieval in recall. This experiment will provide such an evaluation.
FREE ASSOCIATION, FREE RECALL

AND STIMULATED RECALL COMPARED

The standard method of free association presents a test stimulus to which there has been no prior formal training in responding. In free recall, a test is introduced only after the deliberate manipulation of formal laboratory training, but the test itself deliberately omits the use of a stimulus. This differential stress upon the training or test environment should not be allowed to blur the idea that both methods tap memorial processes. In the case of free association, it is memory for materials acquired over the span of time prior to $S$ 's appearance at the laboratory. In the free-recall experiment, $S$ may respond as a function of pre-experimentally or experimentally acquired word-association habits and whichever component is the stronger depends upon $E$. In the case of free recall, $E$ can achieve a purchase upon such behaviors by the selection of training materials which are pre-experimentally associatively related (e.g., Deese, $1959 \mathrm{a}, \mathrm{b}$ ). Where $\mathrm{E}$ deliberately varies the stimuli within the recall environment, proacting pre-experimental associative processes can exest even more powerful influences upon S's recall or misrecall than training variations. This phenomenon was demonstrated by Bilodeau, Fox, \& Blick (1963), who combined features of the methods of free association and free recall, thereby creating the method of stimulated recall. The combination was effected by training $S$ on word material drawn from a pool of $100 R_{2}$ items tabled in the 1954 association norms of Russell and Jenkins (an $R_{2}$ is the second most frequently occurring associate in the hierarchy of free-association responses), and testing for recall of $\mathbf{R}_{\mathbf{2}}$ in the presence of the stimulus term. The essential features of stimulated recall are: (a) a standard laboratory training session, (b) a test instruction to recall, and (c) introduction of stimuli into the test period which are preexperimentally rather than experimentally related to the words of training.

The independent variables defining free association (FA), stimulated recall (SR), and free recall (FR) are outlined in Table 1 along with two important variants. Rows represent the independent variables and columns identify the retention methods. The manipulated variables include (a) prior experimental training or not, (b) type of recall instructions and, (c) presence or absence of stimuli during recall. When stimuli are present in recall, they can be explicitly brought to S's attention (as in standard SR), or as in the case of modified SR (MSR), the recall instructions may make no mention of such stimuli and their employment.

For modified FA (MFA), the operational accent is on the presence of a training period prior to testing. Bilodeau et al

Table 1

Summary of Operations Defining Five Methods of Recall

\begin{tabular}{lllllll} 
& \multicolumn{3}{c}{ Primary Methods } & & \multicolumn{2}{c}{ Modified Methods } \\
\cline { 2 - 4 } Variable & FA & SR & FR & & MFA & MSR \\
\hline Prior Training & No & Yes & Yes & & Yes & Yes \\
Instruction to Recall & No & Yes & Yes & & No $^{1}$ & Yes \\
Test Stimulns & Yes & Yes & No & & Yes & Yes $^{2}$ \\
\hline
\end{tabular}

${ }^{1} N o$ is actually an instruction to free associate. The stressing operations are italicized.

${ }^{2}$ The stimulus, though present, is not called to S's attention. 